\title{
Rheology of hydro-magnetic polymeric material with heat generation/absorption and chemical reaction
}

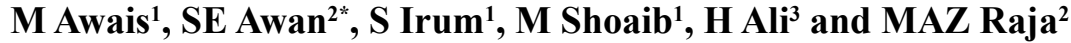 \\ ${ }^{1}$ Department of Mathematics, COMSATS University Islamabad, Attock Campus, Pakistan. \\ ${ }^{2}$ Department of Electrical and Computer Engineering, COMSATS University Islamabad, Attock campus, Pakistan \\ ${ }^{3}$ Department of Electrical Engineering Technology, University of Technology Nowshera, Pakistan.
}

\begin{abstract}
In the present work, the mathematical modelling, transformation and analysis are presented to investigate the magnetohydrodynamic (MHD) flow of a polymeric liquid, i.e., Maxwell fluidic model, over an absorptive wall encapsulated in a medium having porosity. The system model in the form of partial differential equations (PDEs) for fluid flow is converted to a corresponding ordinary differential equations (ODEs)-based system through introduction of dimensionless quantities with the help of appropriate similarity transformation procedures. The velocity, temperature and concentration profiles of the system are calculated for variations in different prominent physical parameters for upper convected Maxwell (UCM) model including combined influences of energy and transfer of mass together with chemical reception and heat generation or absorption as well as Sherwood number, Nusselt number and Deborah number. Lie group analysis was carried out for the nonlinear governing equations and the absolute invariants were found properly. Numerical, as well as analytical computing techniques with the homotopy analysis method (HAM) were utilised to verify and validate the correctness of the approximate solutions for the coupled system. Analysis of the results with exhaustive interpretation for each numerical and graphical illustration further endorsed the effectiveness and worth of the solvers. The trend of fluid flow in terms of streamlines are also presented to show the difference elaboratively between hydromagnetic and hydrodynamic flow situations.
\end{abstract}

Keywords: Heat generation/absorption, lie group analysis, magnetic field effects, MHD, polymeric liquid.

\section{INTRODUCTION}

It is generally acknowledged that non-Newtonian fluid behaviour (categorised by a nonlinear relation between viscosity and strain) can be witnessed in several ordinary and real life fluids including blood, lubricants, paints, foodstuff (including ketchup, cream, cooking oil, butter, etc.), polymers and chemicals. Distant from condensing and retreating properties, some other rheological effects have been observed such as tangential and normal stresses, relaxations and retardation time. Under these features, researchers have proposed fluid models in the past few years. A survey of literature shows that the $1^{\text {st }}$, $2^{\text {nd }}, 3^{\text {rd }}$ and $4^{\text {th }}$ grade differential type fluids have been discussed widely while the slightly non-Newtonian Jeffery fluid, Maxwell fluid, Oldroyd and Burgers fluids are not exploited well as these models are suitable to forecast the relaxation and retardation properties. Harris (1977) proposed mathematical calculations of Maxwell fluid. Several mathematicians and physicists inspected the Maxwell model with different geometries and Zierep and Fetecau (2007) studied dynamic equilibrium equations of 3 Rayleigh-Stokes. They have considered the Stokes problems and discussed the shear stress and dissipation properties. Stability of Maxwell equations have already been examined by Tan and Masuoka (2007). The authors computed the eigenvalue problem,

*Corresponding author (saeed.ehsan@ciit-attock.edu.pk; (iD http://orcid.org/0000-0002-4557-9533) 
and Rayleigh effects, wave effect and frequency for over-stability have been found. Renardy and Renardy (2009) investigated linear solidity of homogeneous flow of the non-Newtonian (upper convected Maxwell) fluid. Kumari and Nath (2009) computed the non-Newtonian upper-convected Maxwell (UCM) fluid past a stagnation point with inclusion of magnetohydrodynamic (MHD). Authors have employed the order analysis to simplify the equations and utilised the finite difference technique for the solution computations. Mass transfer in UCM fluid flow has been analysed by Hayat et al. (2011a). Mathematical modelling for the MHD case has been performed. A correction for MHD Maxwell fluid model equation is presented for the first time in the literature and chemical reaction for the generative and destructive case has been investigated. Effect of 3D flow for UCM fluidic system over a bi-directionally surface with stretching mechanism has been exploited by Hayat et al. (2011b). Mathematical modelling for the three-dimensional case for any rate type fluid has been presented. Deborah effect has been analysed for the axisymmetric, two- and threedimensional flow situations. Unsteady 3D non-Newtonian UCM fluid flow past a smooth linear surface based on linearly stretching in two directions has been presented by Awais et al. (2014). They have modelled the flow of Maxwell fluid for the unsteady case in three-dimensions. Similarity transforms are utilised for simplification and analytical solver homotopy analysis method (HAM), has been invoked for solution construction. Generally in recent past, extensive research work has been conducted to investigate the physical behaviour of fluidic problems (Shahid et al., 2018; Dehghani et al., 2019; Farooq et al., 2019; Fiza et al., 2019; Khan et al., 2019; Kumar et al., 2019; Yasmin et al., 2019; Yousif et al., 2019). The objective of current investigation is to examine the Lie group analysis of UCM fluid flow past an absorptive surface with impact of heat and mass transfer. Generative and destructive chemical receptions along with heat generation or absorption effects are taken into consideration. Outcomes of the velocity, concentration and temperature distribution are computed, and visual representations of residual errors are displayed to show the efficacy of the results. Tabular description has also been performed to show the convergence of the numeric solutions and to present the analogy of obtained results with the already available results in the limiting sense. A graphical presentation has also been given which describes the presentation of sundry measures on velocity, concentration and temperature.

\section{METHODOLOGY}

Methodology is presented in two parts; problem formulation with the help of mathematical formulation is represented in the first part, while Lie group analysis is represented in the second part.

\section{Problem formulation}

The properties of polymeric liquid with electrical conduction past a flat absorptive surface are assumed. Equal forces but in opposite directions are incorporated along with the horizontal axis in such a way that the surface $\bar{y}=0$ is stretched. The magnetic effect $B_{0}$ is along the vertical axis. As shown in Figure 1 the temperature as well as concentration of the fluid at stretched surface with their ambient values are $T_{w}, C_{w}, T_{\infty}$ and $C_{\infty}$ respectively. The system model for the proposed fluid flow is given as follows: (Hayat et al., 2011 a: b; Awais et al., 2016; Saleem et al., 2017)

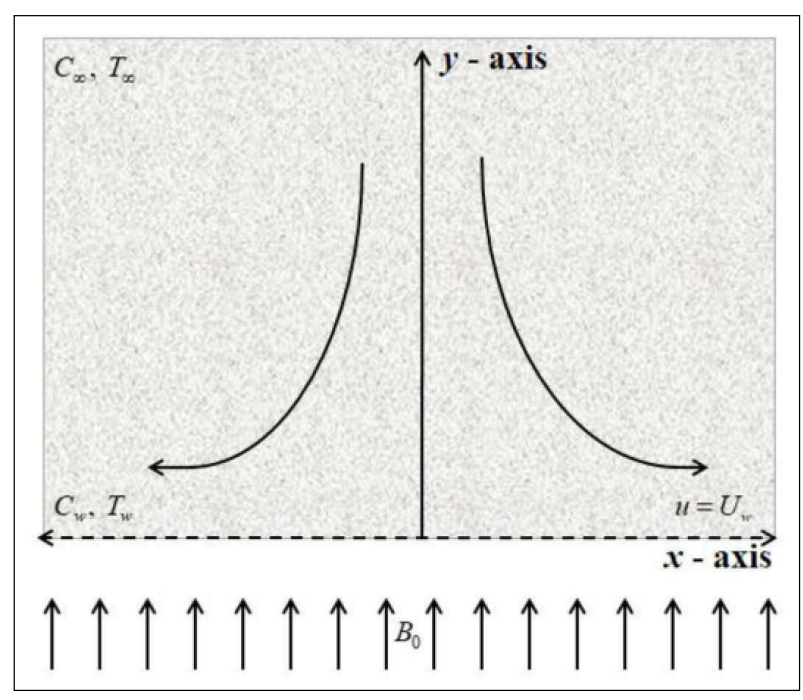

Figure 1: Schematic diagram of flow domain

$\frac{\partial \bar{v}}{\partial \bar{y}}+\frac{\partial \bar{u}}{\partial \bar{x}}=0$

$\bar{v} \frac{\partial \bar{u}}{\partial \bar{y}}+\bar{u} \frac{\partial \bar{u}}{\partial \bar{x}}+\lambda_{1}\left[\bar{u}^{2}\left(\frac{\partial^{2} \bar{u}}{\partial \bar{x}^{2}}\right)+2 \overline{u v}\left(\frac{\partial^{2} \bar{u}}{\partial \bar{x} \partial \bar{y}}\right)+\bar{v}^{2}\left(\frac{\partial^{2} \bar{u}}{\partial \bar{y}^{2}}\right)\right]=$

$\bar{U}_{e} \frac{\partial \bar{U}_{e}}{\partial \bar{x}}+v \frac{\partial^{2} \bar{u}}{\partial \bar{y}^{2}}+\frac{v \tau}{k}\left(\bar{U}_{e}-\bar{u}\right)+\frac{\sigma B_{0}^{2}}{\rho}\left(\bar{U}_{e}-\bar{u}-\lambda_{1} \bar{v} \frac{\partial \bar{u}}{\partial \bar{y}}\right)$,

$\rho\left(\bar{u} \frac{\partial \bar{T}}{\partial \bar{x}}-\alpha \frac{\partial^{2} \bar{T}}{\partial \bar{y}^{2}}+\bar{v} \frac{\partial \bar{T}}{\partial \bar{y}}\right)=\frac{Q_{0}}{C_{p}}\left(\bar{T}-\bar{T}_{\infty}\right)$, 
$\bar{u} \frac{\partial \bar{C}}{\partial \bar{x}}+k_{0}\left(\bar{C}-\bar{C}_{\infty}\right)+\bar{v} \frac{\partial \bar{C}}{\partial \bar{y}}=D \frac{\partial^{2} \bar{C}}{\partial \bar{y}^{2}}$

Equation (1) represents law of conservation of mass, while equation (2) is the law of conservation of momentum. Equation (3) represents the law of conservation of energy and equation (4) is the mass transfer equation.

The wall effects are:

At the surface $\bar{y}=0, \bar{u}=\left(\bar{U}_{w}(\bar{x})\right)=C_{1} \bar{x}, \bar{T}=\left(\bar{T}_{w}\right)$,

$$
\bar{v}=\bar{v}_{w}(\bar{x}), \quad \bar{C}=\bar{C}_{w},
$$

away from the surace $\bar{y} \rightarrow \infty, \quad \bar{u} \rightarrow \bar{U}_{e}(\bar{x})=a \bar{x}$, $\bar{C} \rightarrow\left(\bar{C}_{\infty}\right), \quad \bar{T} \rightarrow \bar{T}_{\infty}$.

Introducing

$$
\begin{gathered}
x=\frac{\bar{x} C_{1}}{U_{1}}, \quad y=\sqrt{\frac{C_{1}}{v}} \bar{y}, \quad u=\frac{\bar{u}}{U_{1}}, \quad v=\frac{\bar{v}}{\sqrt{v C_{1}}}, \\
U_{e}=\frac{\bar{U}_{e}}{U_{1}}, \quad \varphi=\frac{\left(\bar{C}-\bar{C}_{\infty}\right)}{\bar{C}_{w}-\bar{C}_{\infty}}, \quad \theta=\frac{\left(\bar{T}-\bar{T}_{\infty}\right)}{\bar{T}_{w}-\bar{T}_{\infty}} .
\end{gathered}
$$

Substitution of equation (6) into equations (1-5), gives

$$
\begin{aligned}
& \frac{\partial v}{\partial y}+\frac{\partial u}{\partial x}=0 \\
& v\left(\frac{\partial u}{\partial y}\right)+u\left(\frac{\partial u}{\partial x}\right)+\beta\left(u^{2} \frac{\partial^{2} u}{\partial x^{2}}+2 u v \frac{\partial^{2} u}{\partial x \partial y}+v^{2} \frac{\partial^{2} u}{\partial y^{2}}\right)= \\
& U_{e} \frac{\partial U_{e}}{\partial x}+\frac{\partial^{2} u}{\partial y^{2}}+k\left(U_{e}-u\right)+M^{2}\left(-u+U_{e}-(\beta v) \frac{\partial u}{\partial y}\right)
\end{aligned}
$$

$$
v \frac{\partial \theta}{\partial y}+u \frac{\partial \theta}{\partial x}=h s \theta+\frac{1}{\operatorname{Pr}} \frac{\partial^{2} \theta}{\partial y^{2}}
$$

$$
u \frac{\partial \varphi}{\partial x}+v \frac{\partial \varphi}{\partial y}=\frac{1}{S c} \frac{\partial^{2} \varphi}{\partial y^{2}}-\gamma \varphi
$$

$$
u=x, \quad v=\frac{v_{w}}{\sqrt{C_{1} v}}, \quad \theta=1, \quad \varphi=1 \quad \text { at } y=0,
$$$$
u \rightarrow A x, \quad \theta=0, \quad \varphi=0 \quad \text { as } y \rightarrow \infty \text {, }
$$

\section{Lie analysis of the problem}

Introducing

$$
\Gamma=\left\{\begin{array}{c}
x^{*}=x e^{\varepsilon \alpha_{1}}, \quad u^{*}=u e^{\varepsilon \alpha_{4}}, \quad y^{*}=y e^{\varepsilon \alpha_{2}}, \quad \psi^{*}=\psi e^{\varepsilon \alpha_{3}} \\
v^{*}=v e^{\varepsilon \alpha_{5}}, U_{e}^{*}=U_{e} e^{\varepsilon \alpha_{6}}, \quad \theta^{*}=\theta e^{\varepsilon \alpha_{7}}, \varphi^{*}=\varphi e^{\varepsilon \alpha_{8}} .
\end{array}\right.
$$

Subsisting equation (18) into equations (14-17) gives

$$
e^{\varepsilon\left(\alpha_{1}-2 \alpha_{3}+2 \alpha_{2}\right)}\left(\psi_{y^{*}}^{*}, \psi_{y^{*} x^{*}}^{*}-\psi_{x^{*}}^{*} \psi_{y^{*} y^{*}}^{*}\right)+
$$

$\beta e^{\varepsilon\left(2 \alpha_{1}-3 \alpha_{3}+3 \alpha_{2}\right)}\left(\psi_{y^{*}}^{*^{2}} \psi_{x^{*} x^{*} y^{*}}^{*}-2 \psi_{x^{*}}^{*} \psi_{y^{*}}^{*} \psi_{x^{*} y^{*} y^{*}}^{*}+\psi_{x^{*}}^{*} \psi_{y^{*} y^{*} y^{*}}^{*}\right)$ 


$$
\begin{aligned}
& -e^{\varepsilon\left(-2 \alpha_{6}+\alpha_{1}\right)} U_{e}^{*} \frac{\partial U_{e}^{*}}{\partial x^{*}}-e^{\varepsilon\left(-\alpha_{3}+3 \alpha_{2}\right)} \psi \\
& -M^{2}\left(e^{-\varepsilon \alpha_{6}} U_{e}^{*}-e^{\varepsilon\left(-\alpha_{3}+\alpha_{2}\right)} \psi_{y^{*}}^{*}+e^{\varepsilon\left(\alpha_{1}-2 \alpha_{3}+2 \alpha_{2}\right)} \beta \psi_{x^{*}}^{*} \psi_{y^{*} y^{*}}^{*}\right) \\
& -K\left(e^{-\varepsilon \alpha_{6}} U_{e}^{*}-e^{\varepsilon\left(\alpha_{2}-\alpha_{3}\right)} \psi_{y^{*}}^{*}\right)=0
\end{aligned}
$$

$e^{\varepsilon\left(-\alpha_{3}+\alpha_{2}+\alpha_{1}-\alpha_{7}\right)}\left(\psi_{y^{*}}^{*} \theta_{x^{*}}^{*}-\psi_{x^{*}}^{*} \theta_{y^{*}}^{*}\right)-\frac{1}{\operatorname{Pr}} e^{\varepsilon\left(2 \alpha_{2}-\alpha_{7}\right)} \theta_{y^{*} y^{*}}^{*}$

$$
-h s e^{-\varepsilon \alpha_{7}} \theta^{*}=0
$$

$$
e^{\varepsilon\left(-\alpha_{3}+\alpha_{2}+\alpha_{1}-\alpha_{8}\right)}\left(-\psi_{x^{*}}^{*}\left(\varphi_{y^{*}}^{*}\right)+\psi_{y^{*}}^{*}\left(\varphi_{x^{*}}^{*}\right)\right)-
$$$$
\frac{1}{S c} e^{\varepsilon\left(2 \alpha_{2}-\alpha_{8}\right)}\left(\varphi_{y^{*} y^{*}}^{*}\right)+\gamma e^{-\varepsilon \alpha_{8}} \varphi^{*}=0,
$$

$e^{\varepsilon\left(-\alpha_{3}+\alpha_{2}\right)}\left(\psi_{y^{*}}^{*}\right)=\left(e^{-\varepsilon \alpha_{1}}\right) x^{*}, e^{\varepsilon\left(-\alpha_{3}+\alpha_{1}\right)} \psi_{x^{*}}^{*}=-\frac{v_{w}}{\sqrt{C_{1} v}}$,

$$
\theta^{*}=e^{\varepsilon \alpha_{7}}, \varphi^{*}=e^{\varepsilon \alpha_{8}} \text { at } y^{*}=0
$$

$$
e^{\varepsilon\left(-\alpha_{3}+\alpha_{2}\right)}\left(\psi_{y^{*}}^{*}\right) \rightarrow A e^{-\varepsilon \alpha_{1}} x^{*}, \quad \theta^{*}=0, \varphi^{*}=0 \text { as } y^{*} \rightarrow \infty .
$$

The uniformity of the fluidic system is maintained under $\Gamma$, to get

$$
\begin{aligned}
& -3 \alpha_{3}+3 \alpha_{2}+2 \alpha_{1}=-2 \alpha_{3}+2 \alpha_{2}+\alpha_{1}=-2 \alpha_{6}+\alpha_{1}=-\alpha_{3}+3 \alpha_{2}= \\
& -\alpha_{3}+\alpha_{2}=-\alpha_{6},-\alpha_{3}+\alpha_{2}+\alpha_{1}-\alpha_{7}=2 \alpha_{2}-\alpha_{7}=-\alpha_{7} \\
& -\alpha_{3}+\alpha_{2}+\alpha_{1}-\alpha_{8}=2 \alpha_{2}-\alpha_{8}=-\alpha_{8} .
\end{aligned}
$$

After solving we obtain

$$
\alpha_{1}=\alpha_{3}=\alpha_{4}=\alpha_{6} \quad \text { and } \quad \alpha_{2}=\alpha_{8}=\alpha_{7}=\alpha_{5}=0 .
$$

Utilizing equation (24), the proposed transformations $\Gamma$ curtails to

$$
\Gamma=\left\{\begin{array}{lrrr}
x^{*}=x e^{\varepsilon \alpha_{1}}, & u^{*}=u e^{\varepsilon \alpha_{1}}, & y^{*}=y, & \psi^{*}=\psi e^{\varepsilon \alpha_{1}} \\
v^{*}=v, & U_{e}^{*}=U_{e} e^{\varepsilon \alpha_{1}}, & \theta^{*}=\theta, & \varphi^{*}=\varphi .
\end{array}\right.
$$

Using Taylor's method, we obtain

$$
\Gamma=\left\{\begin{array}{cc}
x^{*}=x\left(1+\varepsilon \alpha_{1}\right), & U_{e}^{*}=U_{e}\left(1+\varepsilon \alpha_{1}\right), \quad \psi^{*}-\psi=\psi \varepsilon \alpha_{1}, \\
u^{*}=u\left(1+\varepsilon \alpha_{1}\right), & v^{*}-v=y^{*}-y=\theta^{*}-\theta=\varphi^{*}-\varphi=0 .
\end{array}\right.
$$

In differential form

$\frac{d x}{\alpha_{1} x}=\frac{d \psi}{\alpha_{1} \psi}=\frac{d y}{0}=\frac{d u}{\alpha_{1} u}=\frac{d U_{e}}{\alpha_{1} U_{e}}=\frac{d \theta}{0}=\frac{d v}{0}=\frac{d \varphi}{0}$.

After simplification, we get

$y^{*}=\eta, \quad \psi^{*}=x^{*} f(\eta), \quad U_{e}^{*}=A x^{*}, \quad \theta^{*}=\theta(\eta), \quad \varphi^{*}=\varphi(\eta)$.

Equations (19-22) give

$f^{\prime \prime \prime}-\left(f^{\prime}\right)^{2}+f f^{\prime \prime}+\left(M^{2}+K\right)\left(-f^{\prime}+A\right)-\beta\left((f)^{2} f^{\prime \prime \prime}-2 f f f^{\prime \prime}\right)+$

$M^{2} \beta f f^{\prime \prime}+\lambda^{2}=0$

$\theta^{\prime \prime}+\left(f \theta^{\prime}+h s \theta\right) \operatorname{Pr}=0$

$\varphi^{\prime \prime}+\operatorname{Sc}\left(f \varphi^{\prime}-\gamma \varphi\right)=0$

with wall effects

$\left[\begin{array}{c}f(0)=S(\text { Suctionvelocity }), \quad \varphi(0)=f^{\prime}(0)=1=\theta(0), \text { at surface } \\ f^{\prime}(\infty) \rightarrow A, \quad \theta(\infty) \rightarrow 0, \quad \varphi(\infty) \rightarrow 0, \text { away from surface }\end{array}\right]$

Let $S=-v_{w} / \sqrt{c v}$.

For $\mathrm{S}<0, \mathrm{~S}>0$ represent injection or suction of the system model

Here $N u=-\theta^{\prime}(0)$ and $S h=-\varphi^{\prime}(0)$ are mathematical expressions for local Nusselt and Sherwood numbers, respectively for the proposed fluidic system.

\section{Solution methodology}

The set of equations (29-31) along with the boundary condition given in equation (32) is still a mathematical model and an exact solution does not exist due to the high non-linearity of the proposed fluidic model. For the analytical solution of the proposed fluidic system the homotopy method (13-20) is used.

The following are functions to transform the system

$\left\{\eta^{k} \exp (-n \eta), k \geq 0, n \geq 0\right\}$

For velocity profile

$f_{m}(\eta)=a_{0,0}^{0}+\sum_{n=0}^{\infty} \sum_{k=0}^{\infty}\left(a_{n}^{k} \eta^{k}\right) \mathrm{e}^{-n \eta}$

For temperature profile 


$$
\theta_{m}(\eta)=\sum_{n=0}^{\infty} \sum_{k=0}^{\infty}\left(b_{n}^{k} \eta^{k}\right) \mathrm{e}^{-n \eta},
$$

For concentration profile

$$
\varphi_{m}(\eta)=\sum_{n=0}^{\infty} \sum_{k=0}^{\infty}\left(c_{n}^{k} \eta^{k}\right) \mathrm{e}^{-n \eta}
$$

In the case of HAM, the unique way for choosing the initial approximation is as follows:

Any function that satisfies the boundary condition can be utilised as initial guess in HAM, but surely there are number of functions which satisfy boundary conditions. In that situation we have to choose the initial guess which gives rapid convergence. The initial guess can be found easily with the help of initial operator and boundary conditions.

The initial approximations for velocity profile $f_{0}$, temperature profile $\theta_{0}$ and concentration profile $\varphi_{0}$ are presented as

$$
\begin{aligned}
& f_{0}(\eta)=A \eta+(1-A)\left(1-\mathrm{e}^{-\eta}\right)+S, \\
& \theta_{0}(\eta)=\exp (-\eta) \\
& \varphi_{0}(\eta)=\exp (-\eta)
\end{aligned}
$$

and the operators for the proposed system model are given for

$$
\mathrm{L}_{f}=\frac{d^{3}}{d \eta^{3}}-\frac{d}{d \eta}, \text { for velocity profile }
$$

$\mathbf{L}_{\theta}=\left(\frac{d^{2}}{d \eta^{2}}\right)-1$, for temperature profile

and

$\mathrm{L}_{\varphi}=\left(\frac{d^{2}}{d \eta^{2}}\right)-1$, for concentration profile

Zeroth order deformation for the proposed system model are given by:

For velocity profile

$$
(1-p) \mathbf{L}_{f}\left[f(\eta ; p)-f_{0}(\eta)\right]=p h_{f} N_{f}[f(\eta ; p)] .
$$

For temperature profile

$(1-p) \mathrm{L}_{\theta}\left[\theta(\eta ; p)-\theta_{0}(\eta)\right]=p h_{\theta} N_{\theta}[\theta(\eta ; p), f(\eta ; p)]$. and

$(1-p) \mathbf{L}_{\varphi}\left[\varphi(\eta ; p)-\varphi_{0}(\eta)\right]=p h_{\varphi} N_{\varphi}[\varphi(\eta ; p), f(\eta ; p)]$,

where $p \in[0,1]$ is the embedding parameter for the proposed model.

The boundary conditions will be transformed in the following way:

$$
\left.f(\eta ; p)\right|_{\eta=0}=S,\left.\frac{\partial f}{\partial \eta}(\eta ; p)\right|_{\eta=0}=1,\left.\frac{\partial f}{\partial \eta}(\eta ; p)\right|_{\eta=\infty}=A
$$

$\left.\theta(\eta ; p)\right|_{\eta=0}=1,\left.\theta(\eta ; p)\right|_{\eta=\infty}=0$,

$\left.\varphi(\eta ; p)\right|_{\eta=0}=1,\left.\varphi(\eta ; p)\right|_{\eta=\infty}=0$,

and the non-linear operators $N_{f}, N_{\theta}$ and $N_{\varphi}$ are

$$
\begin{aligned}
& 0=\frac{\partial^{3} f(\eta ; p)}{\partial \eta^{3}}+\beta\left(2 f(\eta ; p) \frac{\partial f(\eta ; p)}{\partial \eta} \frac{\partial^{2} f(\eta ; p)}{\partial \eta^{2}}-\right. \\
& \left.f^{2}(\eta ; p) \frac{\partial^{3} f(\eta ; p)}{\partial \eta^{3}}\right)+A^{2}-\left(M^{2}+K\right)\left(\frac{\partial f(\eta ; p)}{\partial \eta}-A\right)+ \\
& \left(M^{2} \beta+1\right) f(\eta ; p) \frac{\partial^{2} f(\eta ; p)}{\partial \eta^{2}}-\left(\frac{\partial f(\eta ; p)}{\partial \eta}\right)^{2}-N_{f}[f(\eta ; p)]
\end{aligned}
$$

$$
\begin{aligned}
0 & =\frac{\partial^{2} \theta(\eta ; p)}{\partial \eta^{2}}+\operatorname{Pr} f(\eta ; p) \frac{\partial \theta(\eta ; p)}{\partial \eta}+\operatorname{Pr} h s \varphi(\eta ; p) \\
& -N_{\theta}[\theta(\eta ; p), f(\eta ; p)],
\end{aligned}
$$

$N_{\varphi}[\varphi(\eta ; p), f(\eta ; p)]=\frac{\partial^{2} \varphi(\eta ; p)}{\partial \eta^{2}}+S c f(\eta ; p) \frac{\partial \varphi(\eta ; p)}{\partial \eta}$

$$
-\operatorname{Sc} \gamma \varphi(\eta ; p)
$$

Zeroth-order deformation for the proposed fluidic model when $p=0,1$. having solutions in the following forms:

$f_{0}(\eta)=f(\eta ; 0), \quad f(\eta ; 1)=f(\eta)$, for velocity profile

$\theta(\eta ; 0)=\theta_{0}(\eta), \theta(\eta ; 1)=\theta(\eta)$, for concentration profile

...(44) and 
$\varphi(\eta ; 0)=\varphi_{0}(\eta), \varphi(\eta ; 1)=\varphi(\eta)$, for concentration profile

Taylors series methodology is used for simplification of the fluidic model

$f(\eta ; p)-f_{0}(\eta)=\sum_{m=0}^{\infty} f_{m}(\eta) p^{m}$, for velocity profile

$\theta(\eta ; p)-\theta_{0}(\eta)=\sum_{m=0}^{\infty} \theta_{m}(\eta) p^{m}$, for temperature profile

and for concentration profile

$\varphi(\eta ; p)-\varphi_{0}(\eta)=\sum_{m=0}^{\infty} \varphi_{m}(\eta) p^{m}$

$f_{m}(\eta)=\left.\frac{1}{m !} \frac{\partial^{m}}{\partial p^{m}}(f(\eta ; p))\right|_{p=0}, \theta_{m}(\eta)=\left.\frac{1}{m !} \frac{\partial^{m}}{\partial p^{m}}(\theta(\eta ; p))\right|_{p=0}$,

$\varphi_{m}(\eta)=\left.\frac{1}{m !} \frac{\partial^{m}}{\partial p^{m}}(\varphi(\eta ; p))\right|_{p=0}$

The proposed fluidic system given in equations (55-57) is convergent for $p=1$ and expressed as

$f(\eta)-f_{0}(\eta)=\sum_{m=0}^{\infty} f_{m}(\eta)$, for fluidic velocity

$\theta(\eta)=\theta_{0}(\eta)+\sum_{m=0}^{\infty} \theta_{m}(\eta)$, for temperature profile

and

$\varphi(\eta)=\varphi_{0}(\eta)+\sum_{m=0}^{\infty} \varphi_{m}(\eta)$ for concentration profile

The $m^{\text {th }}$ deformation for the system are

$\mathbf{L}_{f}\left[f_{m}(\eta)-\chi_{m} f_{m-1}(\eta)\right]-h_{f} R_{m}^{f}(\eta)=0$,

$\mathbf{L}_{\theta}\left[\theta_{m}(\eta)-\chi_{m} \theta_{m-1}(\eta)\right]-h_{\theta} R_{m}^{\theta}(\eta)=0$,

$\mathrm{L}_{\varphi}\left[\varphi_{m}(\eta)-\chi_{m} \varphi_{m-1}(\eta)\right]-h_{\varphi} R_{m}^{\varphi}(\eta)=0$,

$f_{m}(0)=0, f_{m}^{\prime}(0)=0, \varphi_{m}(0)=0, \theta_{m}(0)=0$ at surface $\theta_{m}(\infty)=0, f_{m}^{\prime}(\infty)=0, \varphi_{m}(\infty)=0$, away from surface.

$$
\begin{aligned}
R_{m}^{f}(\eta)= & f_{m-1}^{\prime \prime \prime}(\eta)-M^{2}\left(f_{m-1}^{\prime}-\beta \sum_{k=0}^{m-1}\left[f_{m-1-k} f_{k}^{\prime \prime}\right]\right) \\
& +\left(1-\chi_{m}\right)\left[A M^{2}+A K+A^{2}\right]+\sum_{k=0}^{m-1}\left[f_{m-1-k} f_{k}^{\prime \prime}\right.
\end{aligned}
$$

$$
\left.-f_{m-1-k}^{\prime} f_{k}^{\prime}\right]-k_{1} f_{m-1}^{\prime}+\beta \sum_{k=0}^{m-1} f_{m-1-k} \sum_{l=0}^{k}\left[2 f_{k-l}^{\prime} f_{l}^{\prime \prime}-f_{k-l} f_{l}^{\prime \prime \prime}\right],
$$

$R_{m}^{\theta}(\eta)=\theta_{m-1}^{\prime \prime}(\eta)+\operatorname{Pr} h s \theta_{m-1}+\operatorname{Pr} \sum_{k=0}^{m-1} \theta_{m-1-k}^{\prime} f_{k}$,

$R_{m}^{\varphi}(\eta)=\varphi_{m-1}^{\prime \prime}(\eta)-S c \gamma \varphi_{m-1}+S c \sum_{k=0}^{m-1} \varphi_{m-1-k}^{\prime} f_{k}$

$\chi_{m}= \begin{cases}0, & m \leq 1, \\ 1, & m>1 .\end{cases}$

\section{RESULTS AND DISCUSSION}

The fluidic model presented in equations (29-31) with the associated boundary condition given in equation (32) are eminently nonlinear coupled differential equations and their exact solution is very much stiff. We employed HAM (Liao, 2009; Awais et al., 2018 a, b; Jangili \& Beg, 2018; Liu and Wang, 2018; Shirkhani et al., 2018; He et al., 2019; Rana et al., 2019) for the solutions construction.

A MATHEMATICA code for HAM has been developed in order to enumerate the solutions of system model shown in equations (66-68) along with associated boundary conditions mentioned in equation (69).

Table 1: Convergence table

\begin{tabular}{llll}
\hline order & $-f^{\prime \prime}(0)$ & $-\varphi^{\prime}(0)$ & $-\theta^{\prime}(0)$ \\
\hline 1 & 1.278240 & 1.260000 & 0.900000 \\
5 & 1.582964 & 1.456694 & 0.777291 \\
10 & 1.592620 & 1.461691 & 0.767614 \\
15 & 1.592768 & 1.461741 & 0.768040 \\
20 & 1.592793 & 1.461742 & 0.768041 \\
25 & 1.592793 & 1.461742 & 0.768041 \\
30 & 1.592793 & 1.461742 & 0.768041 \\
40 & 1.592793 & 1.461742 & 0.768041 \\
50 & 1.592793 & 1.461742 & 0.768041 \\
\hline
\end{tabular}

The convergence of equations (29-31) depends upon auxiliary parameters. Table 1 is arranged to show the convergence analysis of the numerical values. Convergent solutions upto the $6^{\text {th }}$ decimal place are seen. Figures 2 and 3 show the error of the given analysis. Figure 2 shows the absolute error for $\mathrm{K}$ with velocity profile while Figure 3 depicts absolute error for $\mathrm{M}$ with velocity profile. It is clear from Figures 2 and 3 that error in velocity profile for various values of $\mathrm{K}$ and $\mathrm{M}$ is quite negligible and it exist in the range of $10-07$ to $10-09$. 
It is evident from these graphs that the error in current analysis is very much negligible. Tables 2 and 3 provide a comparative performance of the present findings with

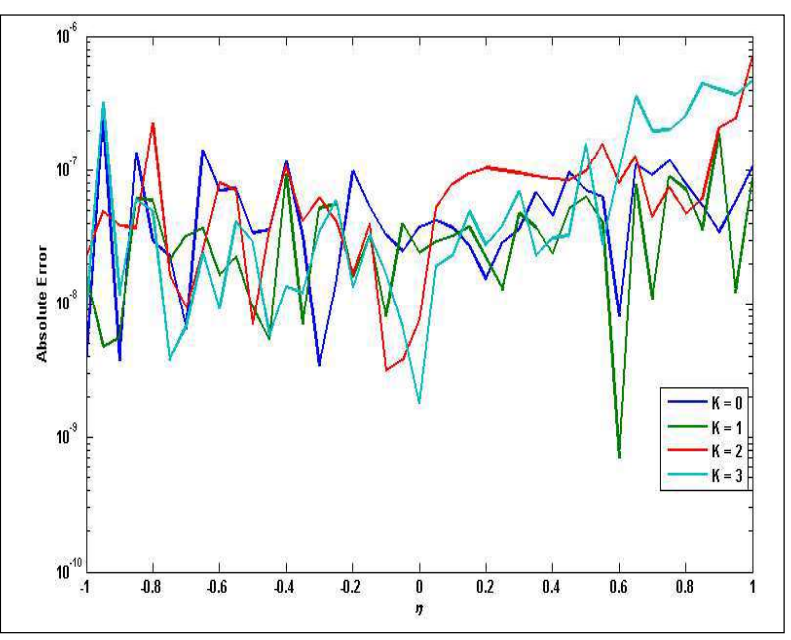

Figure 2: Error for variants of $\mathrm{K}$ with velocity profile

Table 2: Correlation of values of $-\varphi^{\prime}(0)$ when $S=0.5=M=A$, $\mathrm{K}=0.1, \beta=0.0$ and $\mathrm{Sc}=1.0$

\begin{tabular}{ccc}
\hline$\gamma$ & \multicolumn{2}{c}{$-\varphi^{\prime}(0)$} \\
& (Afify \& Elgazery, 2012) & Present results \\
\hline 0.5 & 0.94833 & 0.94836 \\
1.0 & 1.12102 & 1.12102 \\
1.5 & 1.26868 & 1.23865 \\
2.0 & 1.39952 & 1.39978 \\
3.0 & 1.62733 & 1.62729 \\
\hline
\end{tabular}

Streamlines for hydrodynamic and hydro magnetic flow situations are prescribed in Figures 4 and 5. These plots depict that in both flow situations, the streamlines are quite different. Two cases, i.e hydrodynamic $(\mathrm{M}=0)$ and hydromagnetic $(M \neq 0)$ are presented in this analysis. For hydro-magnetic situation the fluid is subjected to a magnetic field, which results in an increase in retarding effects that opposes the flow. In order word effects of magnetic field can be utilised as an agent in the fluid to control the flow very accurately by varying the magnetic field intensity. This behaviour is portrayed in both graphs.

Graphical results (Figures 6-12) are portrayed for different sundry parameters including stagnation point parameter $A$, Deborah number $\beta$, magnetic the already available data. It is seen that the present solution has an acceptable accord with published data (Afify \& Elgazery, 2012).

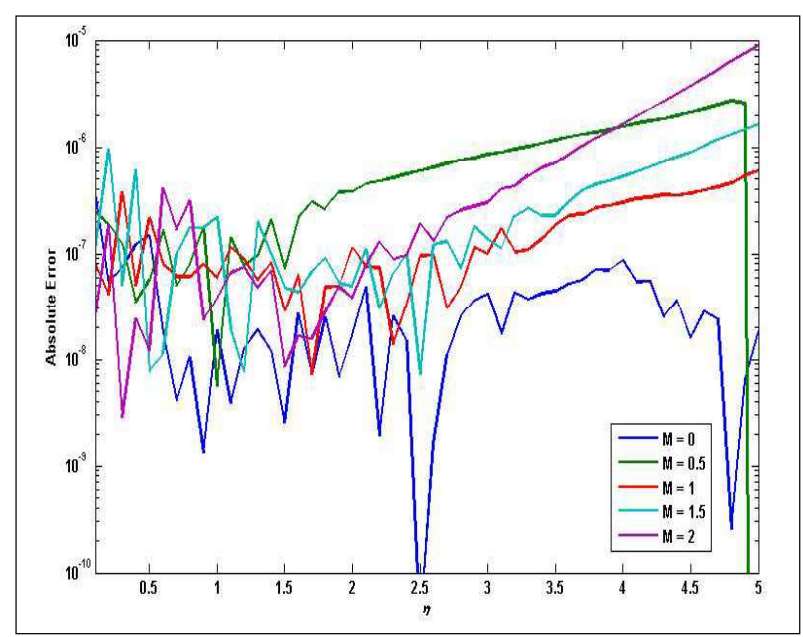

Figure 3: Error for variants of $M$ with velocity profile

Table 3: Comparison of values of $-\theta^{\prime}(0)$ when $\mathrm{S}=0.5=$ $\mathrm{M}=\mathrm{A}, \mathrm{K}=0.1, \beta=0.0$ and $\operatorname{Pr}=1.0$

\begin{tabular}{lcc}
\hline$h s$ & \multicolumn{2}{c}{$-\theta^{\prime}(0)$} \\
& (Afify \& Elgazery, 2012) & Present results \\
\hline-0.2 & 1.13883 & 1.13883 \\
-0.1 & 1.08425 & 1.08425 \\
0.0 & 1.02659 & 1.02659 \\
0.1 & 0.96537 & 0.96537 \\
0.2 & 0.89961 & 0.89961 \\
\hline
\end{tabular}

field parameter $M$, injection/suction parameter $S$ and heat source/sink effect $h s$ and generative/destructive parameter $\gamma$ on the system. It is elucidated from Figure 6 that with an increment in $\mathrm{A}(0<\mathrm{A}<1)$, boundary layer momentum and $f^{\prime}$ increase and for $A=1.0$, the thickness of boundary layer vanishes because the stretching sheet and free stream velocities are equal at $A=1.0$. Moreover for $A>1.0$ the free stream is larger as compared to stretched velocity, which results in an area with high viscosity impact. The Deborah number $\beta$ 's significant impact on $f^{\prime}$ is depicted in Figure 7. Large value of the Deborah number results in the acceleration of momentum boundary layer. It is observed that the layer of boundary momentum and velocity decrease for larger values of Deborah number $\beta$. Figures 8 and 9 present the influence 


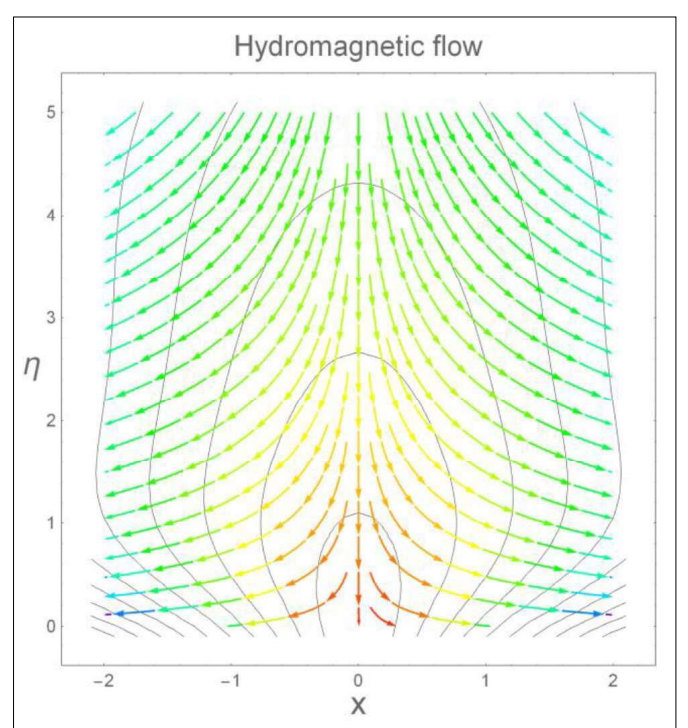

Figure 4: Streamlines for hydrodynamics situation

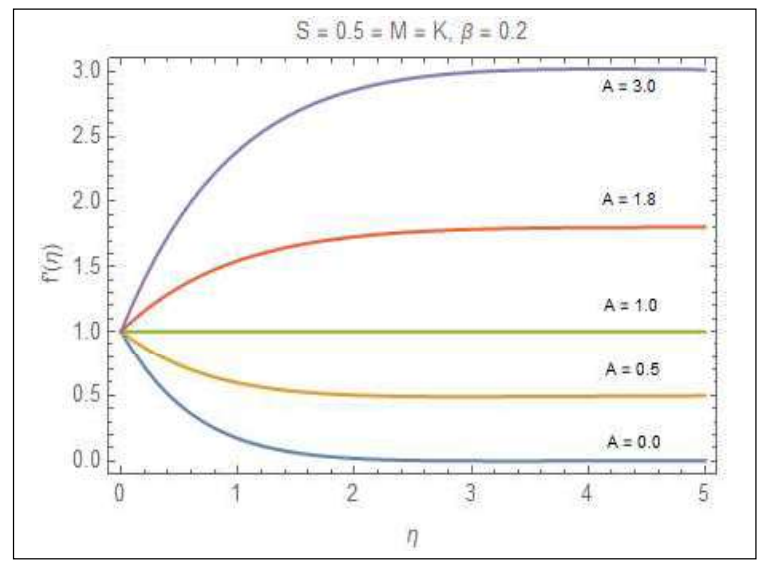

Figure 6: Influence on velocity profile by variation in A

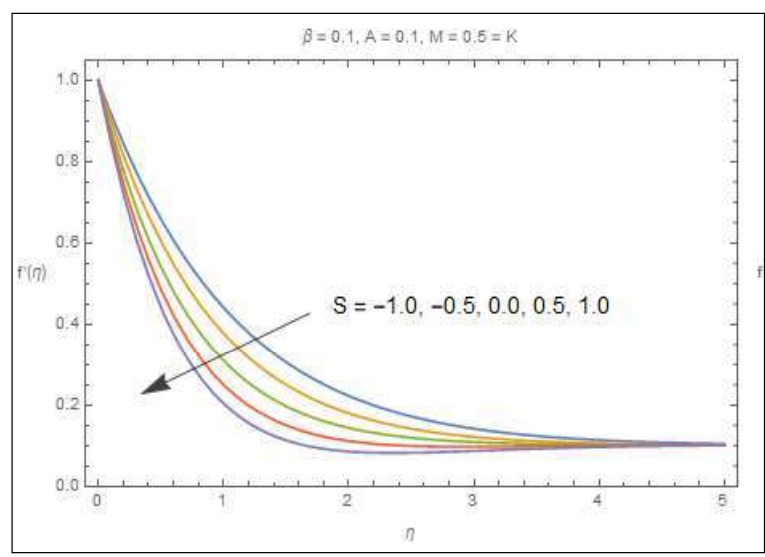

Figure 8: Variation of $\mathrm{S}$ on velocity for $\mathrm{A}<1$

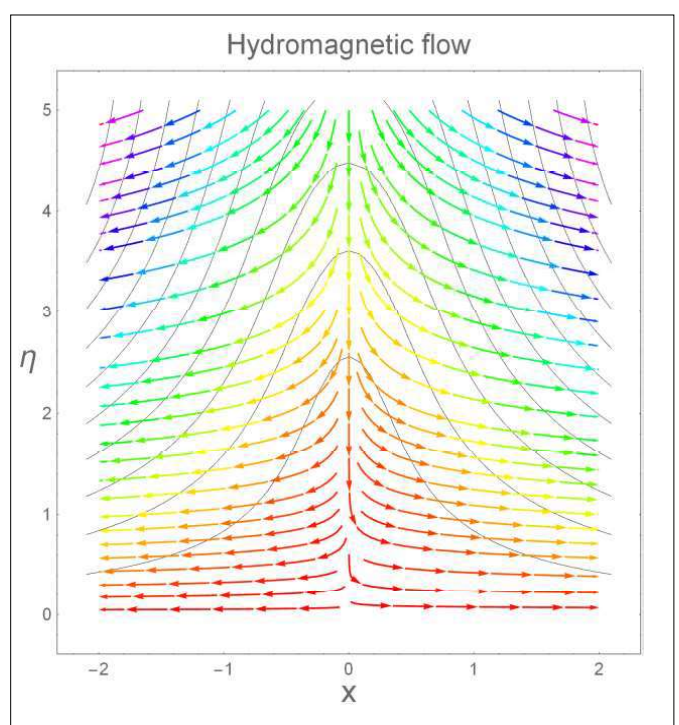

Figure 5: Streamlines for hydro-magnetics situation

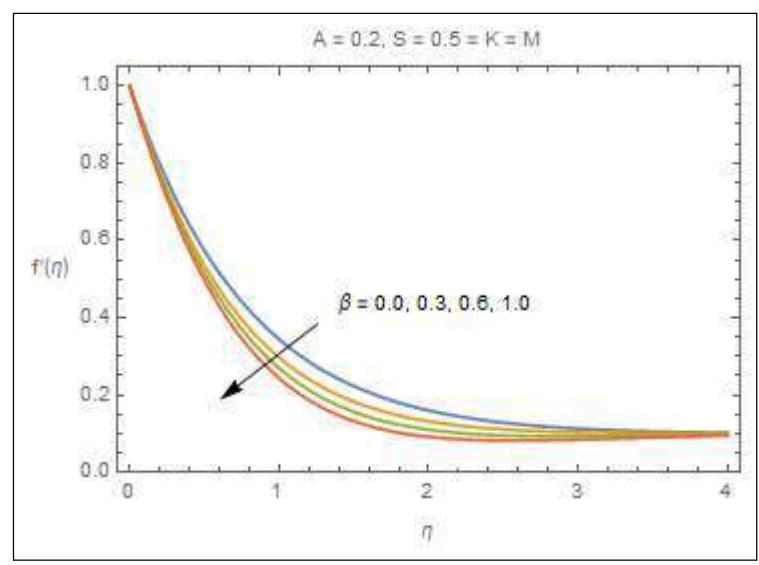

Figure 7: Influence on velocity profile by variation in Deborah number

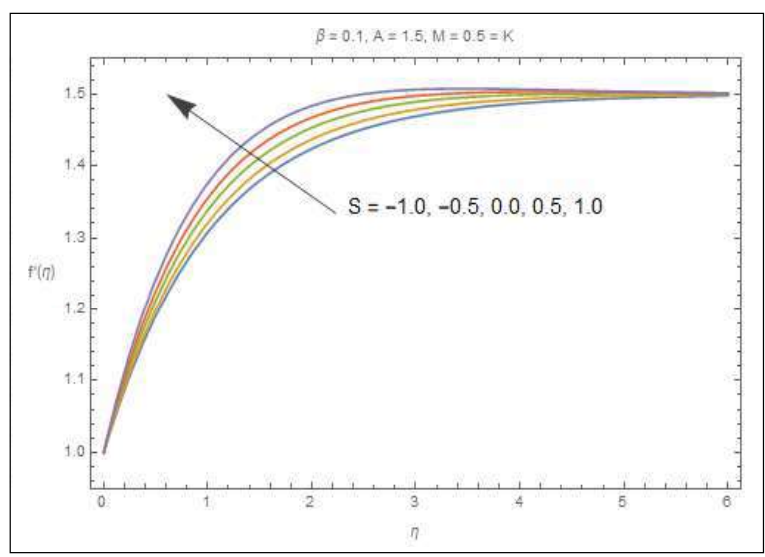

Figure 9: Influence of $\mathrm{S}$ on velocity for $\mathrm{A}>1$ 


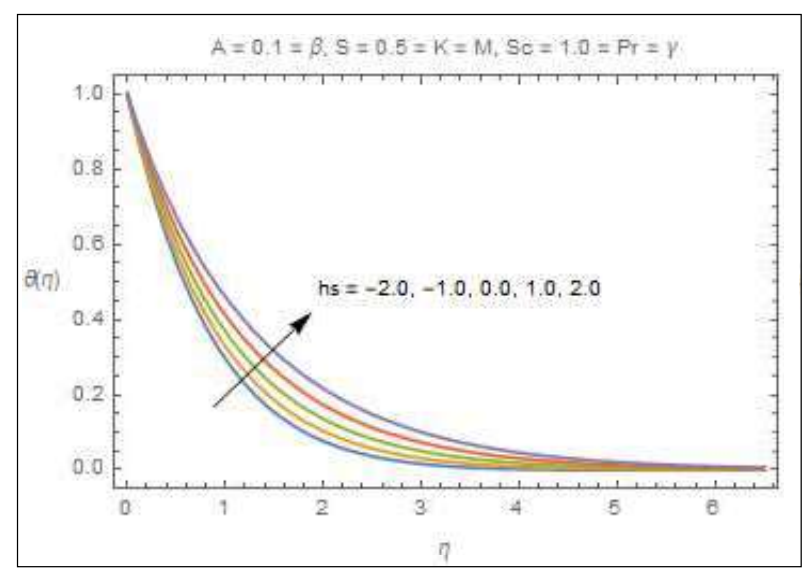

Figure 10: Impact of $h s$ on temperature distribution

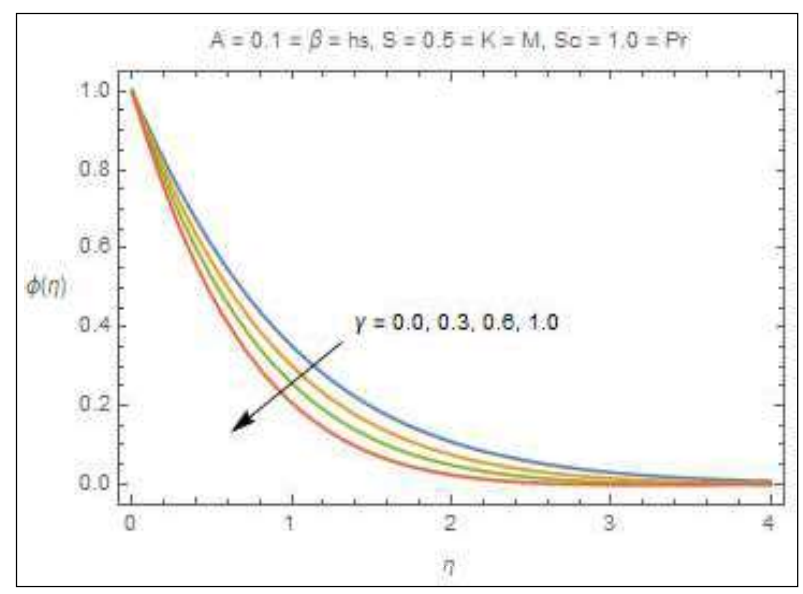

Figure 12: Effects of calamitous chemical reaction of concentration

of suction/injection parameter $S$ for $A<1$ and $A>1$, respectively. For $A<1$ (the stretching velocity dominants over free stream velocity) the decrement in velocity field is observed with an with an increment in $\mathrm{S}$ whereas $A>1$ (mean free stream in dominant) results in an increment in fluidic system velocity profile for greater values of $S$. Moreover, it is also elucidated that $A=1$, which results in vanishing the momentum boundary layer. Figure 10 is provided for the significant impact of $h s$ on temperature. The variation of $h s$ from $-2<h s<2$ is considered and it is seen that the temperature profile increased with an increment in $h s$ from -2 to 2 . Figures 11 and 12 show the properties of destructive/ generative reaction $\gamma$ on $\varphi$. It is observed that diffusion field decreases for destructive $(\gamma$ $>0$ ) and enhances for generative reaction $(\gamma<0)$. $h s$ is the internal heat generation/ absorption phenomenon. For $h s$

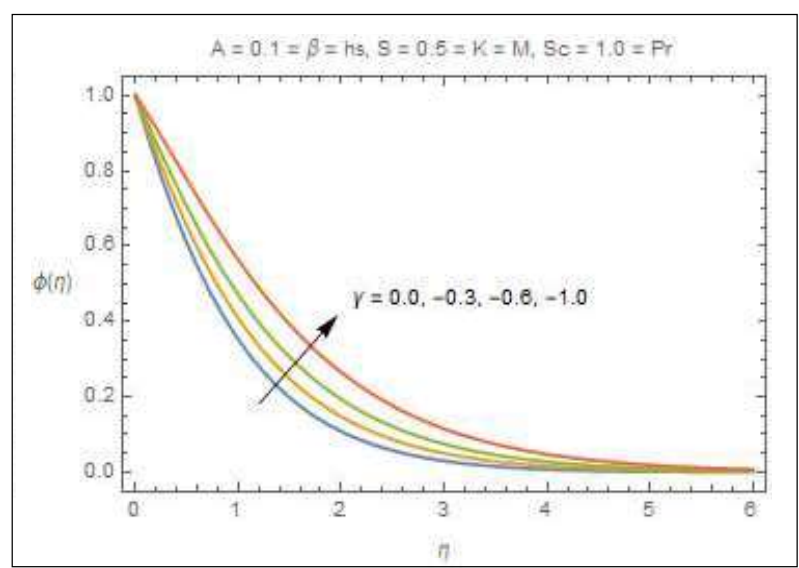

Figure 11: Impact of generative chemical reaction of concentration profile

$<0$, we have the internal heat absorption effect whereas for $h s>0$ we can predict the internal heat generation phenomenon. For $h s=0$, no heat generation/ absorption can be studied.

The role of stochastic numerical computing paradigm (Khan et al., 2015; Mehmood et al., 2018; Awan et al., 2018, Raja et al., 2020, Sabir et al., 2020, Ahmad et al., 2020, Umer et al., 2019, Munir et al., 2019) cannot be denied as an alternate, accurate, reliable and robust computational framework to be exploited in future for solving fluidic systems representing the model for rheology of hydro-magnetic polymeric material with heat generation/absorption and chemical reaction.

\section{CONCLUSIONS}

The current investigation provides an understanding for MHD flow of UCM fluid along with the absorptive surface heated from below including chemical reaction and heat generation or absorption. Lie group analysis is performed and comparison tables are constructed to validate the results available in the literature. The final outcomes of the investigations are listed as follows:

- Increase in Deborah number decelerates the fluid flow and the boundary layer thickness.

- Magnetic field controls the velocity of the fluid very efficiently.

- Increment in heat generation/absorption parameter boosts up the temperature of the fluid.

- Generative and destructive reactions have conflicting effects.

- Sherwood number enhances with $\gamma$ whereas Nusselt number decreases with $h s$. 


\section{REFERENCES}

Afify A.A. \& Elgazery N.S. (2012). Lie group analysis for the effects of chemical reaction on MHD stagnation-point flow of heat and mass transfer towards a heated porous stretching sheet with suction or injection. Nonlinear Analysis: Modelling and Control 17(1): 1-15.

DOI: https://doi.org/10.15388/NA.17.1.14074

Ahmad S.U.I., Faisal F., Shoaib M. \& Raja M.A.Z. (2020). A new heuristic computational solver for nonlinear singular Thomas-Fermi system using evolutionary optimized cubic splines. The European Physical Journal Plus 135: 1-29. DOI: https://doi.org/10.1140/epjp/s13360-019-00066-3

Awais M., Awan S.E., Iqbal K., Khan Z.A. \& Raja M.A.Z. (2018a). Hydromagnetic mixed convective flow over a wall with variable thickness and Cattaneo-Christov heat flux model: OHAM analysis. Results in Physics 8: 621-627. DOI: https://doi.org/10.1016/j.rinp.2017.12.043

Awais M., Hayat T., Muqaddass N., Ali A. \& Awan S.E. (2018b). Nanoparticles and nonlinear thermal radiation properties in the rheology of polymeric material: Results in Physics 8: 1038-1045.

DOI: https://doi.org/10.1016/j.rinp.2018.01.041

Awais M., Hayat T., Alsaedi A. \& Asghar S. (2014). Timedependent three-dimensional boundary layer flow of a Maxwell fluid. Computers and Fluids 91: 21-27.

DOI: https://doi.org/10.1016/j.compfluid.2013.12.002

Awais M., Malik M.Y. \& Awan S.E. (2017). Generalized magnetic effects in a Sakiadis flow of polymeric nanoliquids: analytic and numerical solutions. Journal of Molecular Liquids 241: 570-576.

DOI: https://doi.org/10.1016/j.molliq.2017.06.046

Awais M., Hayat T. \& Ali A. (2016). 3-D Maxwell fluid flow over an exponentially stretching surface using 3-stage Lobatto IIIA formula. AIP Advances 6(5): 055121. DOI: https://doi.org/10.1063/1.4953001

Awan S.E., Khan Z.A., Awais M., Rehman S.U. \& Raja M.A.Z. (2018). Numerical treatment for hydro-magnetic unsteady channel flow of nanofluid with heat transfer. Results in Physics 9: 1543-1554.

DOI: https://doi.org/10.1016/j.rinp.2018.04.068

Dehghani M.S., Toghraie D.S. \& Mehmandoust B. (2019). Mixed-convection nanofluid flow through a grooved channel with internal heat generating solid cylinders in the presence of an applied magnetic field. Heat Transfer Research 50(3): 287-309.

DOI: https://doi.org/10.1615/HeatTransRes.2018025831

Farooq U., Lu D., Munir S., Ramzan M., Suleman M. \& Hussain S. (2019). MHD flow of Maxwell fluid with nanomaterials due to an exponentially stretching surface. Scientific Reports 9(1): Article number 7312.

DOI: https://doi.org/10.1038/s41598-019-43549-0

Fiza M., Islam S., Ullah H. \& Ali Z. (2019). MHD thin film Oldroyd-B fluid with heat and viscous dissipation over oscillating vertical belts. Heat Transfer Research 50(9): 839-849.

DOI: https://doi.org/10.1615/HeatTransRes.2018025031
Harris J. (1977). Rheology and Non-Newtonian Flow. Longman Inc., New York, USA.

Hayat T., Awais M., Qasim M. \&Hendi, A.A. (2011a). Effects of mass transfer on the stagnation point flow of an upperconvected Maxwell (UCM) fluid. International Journal of Heat and Mass Transfer 54(15): 3777-3782.

DOI: https://doi.org/10.1016/j.ijheatmasstransfer.2011.03.003

Hayat T., Awais M. \& Sajid M. (2011b). Mass transfer effects on the unsteady flow of UCM fluid over a stretching sheet. International Journal of Modern Physics B 25(21): 2863-2878.

DOI: https://doi.org/10.1142/S0217979211101375

Hayat T. \& Awais M. (2011). Three-dimensional flow of upperconvected Maxwell (UCM) fluid. International Journal for Numerical Methods in Fluids 66(7): 875-884.

DOI: https://doi.org/10.1002/fld.2289

He S., Sun K. \& Wang H. (2019). Dynamics and synchronization of conformable fractional-order hyperchaotic systems using the homotopy analysis method. Communications in Nonlinear Science and Numerical Simulation 73: 146-164. DOI: https://doi.org/10.1016/j.cnsns.2019.02.007

Jangili S. \& Bég O.A. (2018). Homotopy study of entropy generation in magnetized micropolar flow in a vertical parallel plate channel with buoyancy effect. Heat Transfer Research 49(6): 529-553.

DOI: https://doi.org/10.1615/HeatTransRes.2018018305

Khan A.A., Bukhari S.R., Marin M. \& Ellahi R. (2019). Effects of chemical reaction on third-grade MHD fluid flow under the influence of heat and mass transfer with variable reactive index. Heat Transfer Research 50(11): 1061-1080. DOI: https://doi.org/10.1615/HeatTransRes.2018028397

Khan J.A., Raja M.A.Z., Syam M.I., Tanoli S.A.K. \& Awan S.E. (2015). Design and application of nature inspired computing approach for nonlinear stiff oscillatory problems. Neural Computing and Applications 26(7): 1763-1780. DOI: https://doi.org/10.1007/s00521-015-1841-z

Kumar K.A., Reddy J.R., Sugunamma V. \& Sandeep N. (2019). Simultaneous solutions for MHD flow of Williamson fluid over a curved sheet with nonuniform heat source/sink. Heat Transfer Research 50(6): 581-603.

DOI: https://doi.org/10.1615/HeatTransRes.2018025939

Kumari M. \& Nath G. (2009). Steady mixed convection stagnation-point flow of upper convected Maxwell fluids with magnetic field. International Journal of Non-Linear Mechanics 44(10): 1048-1055.

DOI: https://doi.org/10.1016/j.ijnonlinmec.2009.08.002

Liao S. (2009). Notes on the homotopy analysis method: some definitions and theorems. Communications in Nonlinear Science and Numerical Simulation 14(4): 983-997. DOI: https://doi.org/10.1016/j.cnsns.2008.04.013

Liu J. \& Wang B. (2018). Solving the backward heat conduction problem by homotopy analysis method. Applied Numerical Mathematics 128: 84-97.

DOI: https://doi.org/10.1016/j.apnum.2018.02.002

Mehmood A., Afsar K., Zameer A., Awan S.E. \& Raja M.A.Z. (2019). Integrated intelligent computing paradigm for the dynamics of micropolar fluid flow with heat transfer in a 
permeable walled channel. Applied Soft Computing 79: 139-162.

DOI: https://doi.org/10.1016/j.asoc.2019.03.026

Munir A., Manzar M.A., Khan N.A. \& Raja M.A.Z. (2019). Intelligent computing approach to analyze the dynamics of wire coating with Oldroyd 8-constant fluid. Neural Computing and Applications 31(3): 751-775. DOI: https://doi.org/10.1007/s00521-017-3107-4

Raja M.A.Z., Manzar M.A., Shah S.M. \& Chen Y. (2020). Integrated intelligence of fractional neural networks and sequential quadratic programming for BagleyTorvik systems arising in fluid mechanics. Journal of Computational and Nonlinear Dynamics 15(5): 051003. DOI: https://doi.org/10.1115/1.4046496

Renardy M. \& Renardy Y. (2009). Linear stability of homogeneous elongational flow of the upper convected Maxwell fluid. Journal of Non-Newtonian Fluid Mechanics 160(2): 168-175.

DOI: https://doi.org/10.1016/j.jnnfm.2009.03.011

Rana P., Shukla N., Gupta Y. \& Pop I. (2019). Homotopy analysis method for predicting multiple solutions in the channel flow with stability analysis. Communications in Nonlinear Science and Numerical Simulation 66: 183-193. DOI: https://doi.org/10.1016/j.cnsns.2018.06.012

Sabir Z., Wahab H.A., Umar M., Sakar M.G. \& Raja M.A.Z. (2020). Novel design of Morlet wavelet neural network for solving second order Lane-Emden equation. Mathematics and Computers in Simulation 172: 1-14.

DOI: https://doi.org/10.1016/j.matcom.2020.01.005

Saleem S., Awais M., Nadeem S., Sandeep N. \& Mustafa M.T. (2017). Theoretical analysis of upper-convected Maxwell fluid flow with Cattaneo-Christov heat flux model: Chinese Journal of Physics 55(4): 1615-1625.

DOI: https://doi.org/10.1016/j.cjph.2017.04.005

Shahid A., Bhatti M.M., Bég O.A. \& Kadir A. (2018).
Numerical study of radiative Maxwell viscoelastic magnetized flow from a stretching permeable sheet with the Cattaneo-Christov heat flux model. Neural Computing and Applications 30(11): 3467-3478.

DOI: https://doi.org/10.1007/s00521-017-2933-8

Shirkhani M.R., Hoshyar H.A., Rahimipetroudi I., Akhavan H. \& Ganji D.D. (2018). Unsteady time-dependent incompressible Newtonian fluid flow between two parallel plates by homotopy analysis method (HAM), homotopy perturbation method (HPM) and collocation method (CM). Propulsion and Power Research 7(3): 247-256.

DOI: https://doi.org/10.1016/j.jppr.2018.07.005

Tan W. \& Masuoka T. (2007). Stability analysis of a Maxwell fluid in a porous medium heated from below. Physics Letters A 360(2): 454-460.

DOI: https://doi.org/10.1016/j.physleta.2006.08.054

Umar M., Sabir Z. \& Raja M.A.Z. (2019). Intelligent computing for numerical treatment of nonlinear prey-predator models. Applied Soft Computing 80: 506-524.

DOI: https://doi.org/10.1016/j.asoc.2019.04.022

Yasmin H., Farooq S., Awais M., Alsaedi A. \& Hayat T. (2019). Significance of heat and mass process in peristalsis of a rheological material. Heat Transfer Research 50(16): 1561-1580.

DOI: https://doi.org/10.1615/HeatTransRes.2019026167

Yousif M.A., Ismael H.F., Abbas T. \& Ellahi R. (2019). Numerical study of momentum and heat transfer of MHD Carreau nanofluid over an exponentially stretched plate with internal heat source/sink and radiation. Heat Transfer Research 50(7): 649-658.

DOI: https://doi.org/10.1615/HeatTransRes.2018025568

Zierep J. \& Fetecau C. (2007). Energetic balance for the Rayleigh-Stokes problem of a Maxwell fluid. International Journal of Engineering Science 45(2): 617-627. DOI: https://doi.org/10.1016/j.ijengsci.2007.04.015 